\section{Avaliação de desempenho da regionalização da vigilância em saúde em seis Regiões de Saúde brasileiras}

\author{
Evaluation of the performance of regionalization \\ of health surveillance in six Brazilian \\ Health Regions
}

\section{Evaluación de desempeño de la regionalización de la vigilancia en salud en seis Regiones de Salud brasileñas}

\section{Resumo}

O objetivo deste estudo foi avaliar o desempenho da regionalização da vigilância em saúde em seis Regiões de Saúde com diferentes níveis de desenvolvimento no Brasil. Adotou-se uma abordagem quantitativa transversal e os dados foram coletados utilizando-se um questionário estruturado, elaborado com base nas três dimensões da pesquisa (Politica, Estrutura e Organização), aplicado a 31 atores-chave da vigilância em saúde das Regiões de Saúde e dos municípios selecionados. Utilizou-se, como tendência central, o escore médio, e para cada dimensão e atributo foram construídos indices sintéticos. Atribuíram-se três pontos de corte para avaliação do desempenho: valores iguais ou abaixo de 4,99 foram considerados insatisfatórios; entre 5,00 e 6,99, intermediários; e iguais ou acima de 7,00, satisfatórios. $O$ estudo evidenciou que o desempenho da regionalização da vigilância em saúde foi considerado satisfatório apenas em uma região, intermediário em quatro e insatisfatório também em uma. Entre as três dimensões, os melhores desempenhos foram da Política e da Organização, e o mais frágil, da Estrutura. Conclui-se que, em geral, quanto maior o nível de desenvolvimento socioeconômico e da oferta de serviços, melhor o desempenho da regionalização da vigilância em saúde. A avaliação apresentada retrata a complexidade de contextos de diferentes regiões brasileiras, o que contribui para o entendimento da dinâmica da regionalização da vigilância em saúde no país.

Regionalização; Vigilância em Saúde Pública; Avaliação em Saúde
Ana Coelho de Albuquerque 1,2 Eduarda Ângela Pessoa Cesse 1 Eronildo Felisberto 2 Isabella Chagas Samico 2 Paulo Germano de Frias 2

doi: 10.1590/0102-311X00065218

\author{
Correspondência \\ A. C. Albuquerque \\ Instituto Aggeu Magalhães, Fundação Oswaldo Cruz. \\ Av. Professor Moraes Rego s $/ n$, Cidade Universitária, Recife, PE \\ 50740-465, Brasil. \\ ana_albuquerque1@hotmail.com \\ 1 Instituto Aggeu Magalhães, Fundação Oswaldo Cruz, Recife, \\ Brasil. \\ 2 Grupo de Estudos de Gestão e Avaliação em Saúde, Instituto de \\ Medicina Integral Professor Fernando Figueira, Recife, Brasil.
}




\section{Introdução}

A organização do Sistema Único de Saúde (SUS) baseia-se em um modelo pautado na integração interfederativa, na regionalização e na hierarquização dos serviços, que está disposto na Constituição brasileira. Embora se reconheça que a Carta Magna contribuiu para avanços na ampliação do acesso a serviços públicos, para a reestruturação do modelo de atenção e para a melhoria de indicadores de saúde, a implantação do SUS explicitou contradições entre agendas: de um lado, a universalização da saúde, de outro, a reforma do Estado de inspiração liberal nos anos subsequentes 1.

A partir da década de 1990, a descentralização político-administrativa do SUS centrada na municipalização 2, ao ampliar as atribuições municipais na provisão de serviços e ao desconsiderar o papel dos estados, favoreceu a inflexão do processo de regionalização ${ }^{3}$. Por outro lado, a fragilidade no planejamento da descentralização comprometeu sua adequação às múltiplas realidades brasileiras ao desconsiderar as condições políticas, administrativas, técnicas, financeiras e de necessidades de saúde dos municípios, visando à redução das desigualdades. Apenas nos anos 2000 a regionalização ganhou relevo, e a definição de Região de Saúde apareceu pela primeira vez na Norma Operacional da Assistência à Saúde (NOAS), cujo principal objetivo era a equidade na alocação de recursos e no acesso às ações e serviços de saúde 3 , relevante ante as desigualdades que demarcam o território brasileiro 4 .

Diante do complexo cenário demográfico e epidemiológico nacional, caracterizado pelo rápido envelhecimento populacional associado a elevadas taxas de morbimortalidade por doenças crônicas e causas externas, sobrepostas às infecciosas e parasitárias, com níveis diferenciados de transição entre grupos sociais 5,6, impuseram-se transformações nas respostas sanitárias, inclusive na forma como o sistema de saúde se organiza para ofertar serviços de assistência e vigilância 6,7. A ampliação do escopo de atuação da vigilância em saúde em decorrência desses desafios, além da mobilidade das pessoas em busca de assistência e de melhores condições de vida, influenciou sua estrutura organizacional e gerou necessidades de respostas por vezes não viáveis aos municípios.

A legislação brasileira vigente compreende a vigilância em saúde como um processo sistemático que envolve desde a coleta e análise até a disseminação de dados sobre eventos relacionados com a saúde, articulando saberes e práticas relativas às vigilâncias: epidemiológica, ambiental, do trabalhador e sanitária, com vistas à implementação de políticas públicas de promoção da saúde, proteção, prevenção e controle de riscos, agravos e doenças 8 . Esse conceito foi sendo ressignificado e o lançamento, em 2018, da Política Nacional de Vigilância em Saúde (PNVS) reflete o esforço e os debates, nem sempre consensuais, quanto à necessidade de reorganização da vigilância em saúde na perspectiva de um sistema de saúde universal, integral e equânime 9,10 .

Dentre as estratégias para a organização da vigilância em saúde, destacam-se: a articulação entre as vigilâncias; a integração com a rede de atenção à saúde; os sistemas de informação integrados; a gestão do trabalho; a educação permanente; os estudos e pesquisas; a comunicação e o controle social, associado à regionalização das ações e serviços de vigilância em saúde ${ }^{9}$. No entanto, apesar da publicação da PNVS, os atos normativos do Ministério da Saúde sobre a vigilância em saúde não preveem a reorganização de serviços e ações na perspectiva regionalizada, embora se depreenda o seu papel na consolidação da regionalização 8,11,12,13. Ressalta-se que compete ao Sistema Nacional de Vigilância em Saúde e ao Sistema Nacional de Vigilância Sanitária a execução dessas ações e serviços relacionados com a política 9 .

Inúmeros são os estudos sobre avaliação da vigilância em saúde 14,15,16,17,18,19, entretanto, ainda são escassos os que abordam o tema sobre a estruturação da regionalização e o seu desempenho. Não há consenso quanto à melhor abordagem para aferir o desempenho de sistemas de saúde, em parte pelo seu caráter contingente, variável conforme valores, princípios e concepções sobre saúde, aliado à pluralidade conceitual e metodológica dos modelos avaliativos 20,21,22. Apesar disso, a avaliação de desempenho é apontada como propulsora de mudanças e promotora de maior responsabilização, transparência e qualidade 23,24 .

No Brasil, as avaliações de desempenho da vigilância em saúde utilizaram diferentes abordagens metodológicas: da análise da conformidade 15 a modelos multidimensionais 19 , com o uso de dados primários 15, secundários 14 ou ambos 19, e diferentes recortes geográficos. Entretanto, nenhum deles adotou a perspectiva regional. A diversidade de estratégias e modelos adotados sinaliza a busca de 
abordagens adequadas para a avaliação do desempenho da vigilância em saúde, considerando seu estágio de desenvolvimento e as necessidades prementes 19.

Diante dos desafios que a vigilância em saúde enfrenta ao considerar as complexas inter-relações do processo saúde/doença 25 e a necessidade de um sistema mais efetivo, em particular nas Regiões de Saúde pelo insuficiente acúmulo gerencial para este nível organizacional 26, este artigo tem por objetivo avaliar o desempenho da regionalização da vigilância em saúde em Regiões de Saúde com diferentes níveis de desenvolvimento no Brasil.

\section{Método}

Estudo integrante da pesquisa Politica, Planejamento e Gestão das Regiões e Redes de Atenção à Saúde no Brasil - Região e Redes 27, em que se realizou uma avaliação do desempenho da regionalização da vigilância em saúde em seis regiões, com base na abordagem quantitativa transversal.

A seleção das unidades de análise partiu das 436 Regiões de Saúde brasileiras, fundamentada em tipologia previamente desenvolvida 28 , que adotou a análise de cluster para o desenvolvimento socioeconômico e a oferta e complexidade dos serviços de saúde no contexto regional como condições estruturais de regionalização. Identificaram-se cinco grupos: (1) baixo desenvolvimento socioeconômico e baixa oferta de serviços; (2) médio/alto desenvolvimento socioeconômico e baixa oferta; (3) médio desenvolvimento socioeconômico e média oferta de serviços; (4) alto desenvolvimento socioeconômico e média oferta de serviços; e (5) alto desenvolvimento socioeconômico e alta oferta de serviços.

De cada grupo foi selecionada uma região e agregada uma interestadual por suas características particulares, de Unidade Federada diferente, totalizando seis regiões; e em cada uma delas foram selecionados 3 a 4 municípios em uma amostra não probabilística, incluindo o município-sede e os demais escolhidos por conveniência, perfazendo um total de 21, descritos na Tabela 1. Os dados da Região de Saúde I (RS I) referentes à V Diretoria Regional de Saúde do Estado de São Paulo não separaram Barretos Norte e Sul, devido ao número de respondentes, e na região interestadual, Rede Interestadual de Atenção à Saúde do Vale Médio do São Francisco (Petrolina, Pernambuco e Juazeiro, Bahia - PEBA), os dados foram apresentados separadamente para a RS V (VIII Região de Saúde de Pernambuco) e RS VI (Núcleo Regional de Saúde Norte, Bahia), pois o arranjo organizacional da vigilância em saúde nesta região difere da assistência (Tabela 1).

Para a análise do desempenho da regionalização foram consideradas as dimensões: Política, Estrutura e Organização, categorizadas em atributos, conforme descrito na Tabela 2.

Baseando-se nos atributos de cada dimensão, pesquisadores de 18 instituições de todas as macrorregiões brasileiras elaboraram um questionário estruturado para apreender o desempenho da regionalização da vigilância em saúde 29 , posteriormente validado por especialistas, sendo 15 da vigilância em saúde e cinco da avaliação em saúde, conforme técnica previamente descrita 29 . As questões contidas no instrumento foram expressas majoritariamente na escala de Likert, embora também contenham algumas dicotômicas e multinomiais. O questionário foi aplicado a gestores e a profissionais da vigilância em saúde ou, na sua ausência, da vigilância epidemiológica, ambiental e sanitária, conjuntamente, totalizando 31 respondentes, sendo 25 municipais e seis regionais. Os questionários foram aplicados entre agosto de 2015 e junho de 2016.

Para as questões expressas na escala de Likert calculou-se o escore médio (EM), em que o valor um (1) equivale à pior avaliação; e o cinco (5), à melhor em relação à questão investigada. Para as questões dicotômicas e multinomiais utilizou-se o percentual das respostas afirmativas e/ou positivas em relação ao item investigado. O índice de cada dimensão foi obtido por meio de média aritmética baseando-se na padronização das questões em uma escala de 1 a 10 pontos. Para cada atributo foram construídos índices sintéticos, expressos na mesma escala, compostos pela média dos resultados das variáveis. A classificação do desempenho de cada região, dimensão e atributo considerou insatisfatórios valores iguais ou abaixo de 4,99; intermediários, entre 5,00 e 6,99; e satisfatórios, iguais ou acima de 7,00. Utilizou-se o software Excel (https://products.office.com/) para as tabulações e o programa SPSS para Windows, versão 21.0 (https://www.ibm.com/), para a análise estatística. 
Tabela 1

Regiões de Saúde pesquisadas segundo tipologia * e municípios selecionados para o estudo. Brasil, 2017.

\begin{tabular}{|c|c|c|c|}
\hline Região de Saúde & Tipologia & Municípios da Região de Saúde & $\begin{array}{l}\text { Municípios selecionados } \\
\text { para o estudo }\end{array}$ \\
\hline $\begin{array}{l}\text { V Diretoria Regional de } \\
\text { Saúde (São Paulo) }\end{array}$ & Grupo 5 & $\begin{array}{c}\text { Altair, Barretos, Bebedouro, Cajobi, Colina, Colômbia, Guaíra, Guaraci, } \\
\text { Jaborandi, Monte Azul, Paulista, Olímpia, Severínia, Taiaçu, Taiúva, } \\
\text { Taquaral, Terra Roxa, Viradouro e Vista Alegre do Alto. }\end{array}$ & $\begin{array}{l}\text { Barretos (Sede), Olímpia, } \\
\text { Bebedouro, Cajobi e } \\
\text { Taiúva. }\end{array}$ \\
\hline $\begin{array}{l}\text { Região de Saúde do entorno } \\
\text { de Manaus e Alto Rio Negro } \\
\text { (Amazonas) }\end{array}$ & Grupo 4 & $\begin{array}{c}\text { Manaus, Presidente Figueiredo, Rio Preto de Eva, Iranduba, Careiro de } \\
\text { Várzea, Careiro Castanho, Manaquiri, Autazes, Nova Olinda do Norte, São } \\
\text { Gabriel da Cachoeira, Santa Isabel do Rio Negro e Barcelos. }\end{array}$ & $\begin{array}{l}\text { Manaus (Sede), Careiro } \\
\text { da Várzea e São Gabriel } \\
\text { da Cachoeira. }\end{array}$ \\
\hline $\begin{array}{l}\text { Região de Saúde da Baixada } \\
\text { Cuiabana (Mato Grosso) }\end{array}$ & Grupo 4 & $\begin{array}{c}\text { Barão de Melgaço, Chapada dos Guimarães, Jangada, Nossa Sra. } \\
\text { Livramento, Nova Brasilândia, Planalto da Serra, Poconé, Santo Antônio } \\
\text { do Leverger, Cuiabá, Várzea Grande e Acorizal. }\end{array}$ & $\begin{array}{l}\text { Cuiabá (Sede), Várzea } \\
\text { Grande e Acorizal. }\end{array}$ \\
\hline $\begin{array}{l}\text { Região de Saúde } 9 \text { - } \\
\text { Carbonífera/Costa Doce (Rio } \\
\text { Grande do Sul) }\end{array}$ & Grupo 3 & $\begin{array}{l}\text { Arambaré, Arroio dos Ratos, Barão do Triunfo, Barra do Ribeiro, Butiá, } \\
\text { Camaquã, Cerro Grande do Sul, Charqueadas, Chuvisca, Dom Feliciano, } \\
\text { Eldorado do Sul, General Câmara, Guaíba, Mariana Pimentel, Minas do } \\
\text { Leão, São Jerônimo, Sentinela do Sul, Sertão Santana e Tapes. }\end{array}$ & $\begin{array}{l}\text { Guaíba (Sede), Camaquã, } \\
\text { Dom Feliciano e Sertão } \\
\text { Santana. }\end{array}$ \\
\hline $\begin{array}{l}\text { VIII Região de Saúde } \\
\text { (Pernambuco) }\end{array}$ & Grupo 2 & $\begin{array}{l}\text { Petrolina, Afrânio, Cabrobó, Dormentes, Lagoa Grande, Orocó e Santa } \\
\text { Maria da Boa Vista. }\end{array}$ & $\begin{array}{l}\text { Petrolina (Sede), Cabrobó } \\
\text { e Orocó. }\end{array}$ \\
\hline $\begin{array}{l}\text { Núcleo Regional de Saúde } \\
\text { Norte (Bahia) }\end{array}$ & Grupo 1 & $\begin{array}{l}\text { Juazeiro, Campo Alegre de Lourdes, Canudos, Casa Nova, Curaçá, Pilão } \\
\text { Arcado, Remanso, Sento Sé, Sobradinho e Uauá. }\end{array}$ & $\begin{array}{l}\text { Juazeiro (Sede), Remanso } \\
\text { e Sobradinho. }\end{array}$ \\
\hline
\end{tabular}

* De acordo com Viana et al. 28.

Tabela 2

Dimensões e atributos para a avaliação do desempenho da regionalização da vigilância em saúde. Brasil, 2017.

\begin{tabular}{|c|c|c|}
\hline Dimensão & Atributo & Descrição do atributo \\
\hline \multirow[t]{3}{*}{ Política } & Protagonismo da & Referente à importância dos: órgãos públicos de organização do sistema de saúde, organizações da \\
\hline & Região de Saúde & sociedade civil, instituições acadêmicas e conselhos participativos. \\
\hline & Importância da CIR & Referente à importância da CIR na discussão de temáticas referentes à vigilância em saúde. \\
\hline \multirow[t]{4}{*}{ Estrutura } & $\begin{array}{l}\text { Mobilização de apoios } \\
\text { para as ações }\end{array}$ & Mobilização de apoio para ações de vigilância em saúde. \\
\hline & Suficiência financeira & $\begin{array}{l}\text { Participação das esferas municipal, estadual e federal no custeio da vigilância em saúde; percentual de } \\
\text { execução dos recursos do Piso Fixo e Variável da Vigilância e Promoção da Saúde; investimento para a } \\
\text { vigilância em saúde na região. }\end{array}$ \\
\hline & $\begin{array}{l}\text { Suficiência de recursos } \\
\text { humanos }\end{array}$ & Suficiência de recursos humanos por categorias profissionais. \\
\hline & $\begin{array}{l}\text { Suficiência de recursos } \\
\text { físicos }\end{array}$ & $\begin{array}{l}\text { Suficiência de estrutura; mobilização de insumos, medicamentos e exames para os programas de } \\
\text { controle da tuberculose e dengue. }\end{array}$ \\
\hline \multirow[t]{3}{*}{ Organização } & Planejamento e gestão & $\begin{array}{l}\text { Referente aos parâmetros utilizados no planejamento da vigilância em saúde; definição de práticas e } \\
\text { processos de trabalhos e contribuição da gestão. }\end{array}$ \\
\hline & Integração & $\begin{array}{c}\text { Integração inter e intrassetorial; mecanismos de integração da vigilância em saúde com a atenção } \\
\text { primária à saúde. }\end{array}$ \\
\hline & $\begin{array}{l}\text { Controle de } \\
\text { determinantes, riscos e } \\
\text { danos à saúde }\end{array}$ & $\begin{array}{l}\text { Frequência de doenças de notificação compulsória encerradas por critério laboratorial; frequência de } \\
\text { envio de amostras laboratoriais de exames de interesse para vigilância em saúde (análises laboratoriais } \\
\text { de interesse das vigilâncias epidemiológica, ambiental e sanitária) para análise em outra Região de Saúde; } \\
\text { realização de ações de controle da tuberculose; consolidação e análise dos dados gerados pelos Sistemas } \\
\text { de Informação (SIM, SINAN etc.); acompanhamento dos casos de tuberculose resistente e multidroga } \\
\text { resistente; realização de ações de controle do dengue. }\end{array}$ \\
\hline
\end{tabular}

CIR: Comissão Intergestores Regional; SIM: Sistema de Informações sobre Mortalidade; SINAN: Sistema de Informações de Agravos de Notificação. 
Para a compreensão de aspectos estruturais e organizacionais, foram utilizadas o dengue e a tuberculose como condições traçadoras ${ }^{30}$, por apresentarem alta prevalência e disseminação em todo o território nacional, explicitação quanto aos critérios normativos para seu diagnóstico e tratamento, e à existência de programas de prevenção e controle com ações e fluxos determinados entre as instâncias gestoras.

O trabalho foi aprovado pelo Comitê de Ética em Pesquisa em Seres Humanos da Faculdade de Medicina, Universidade de São Paulo (FM/USP), com o número de protocolo 071/2015.

\section{Resultados}

O desempenho da regionalização da vigilância em saúde foi satisfatório apenas na RS I (EM 7,56), insatisfatório na RS II (EM 4,75) e intermediário nas demais, RS III (EM 6,70), RS IV (EM 6,21), RS V (EM 6,95) e RS VI (EM 6,32). Em geral, entre as três dimensões que influenciam o processo de regionalização, os melhores desempenhos foram da Política e da Organização; e o mais frágil, da Estrutura (Tabela 3).

\section{Tabela 3}

Índice de desempenho da regionalização da vigilância em saúde e classificação, segundo dimensões e atributos por Região de Saúde. Brasil, 2017.

\begin{tabular}{|c|c|c|c|c|c|c|}
\hline \multirow[t]{3}{*}{ Atributos/Dimensões } & \multicolumn{6}{|c|}{ Região de Saúde * } \\
\hline & I & II & III & IV & $\mathbf{v}$ & VI \\
\hline & \multicolumn{6}{|c|}{ Índice de desempenho/Classificação } \\
\hline Protagonismo da região & $\begin{array}{c}7,15 / \\
\text { Satisfatório }\end{array}$ & $\begin{array}{c}5,41 / \\
\text { Intermediário }\end{array}$ & $\begin{array}{c}\text { 6,91/ } \\
\text { Intermediário }\end{array}$ & $\begin{array}{c}\text { 6,85/ } \\
\text { Intermediário }\end{array}$ & $\begin{array}{c}\text { 7,45/ } \\
\text { Satisfatório }\end{array}$ & $\begin{array}{c}5,88 / \\
\text { Intermediário }\end{array}$ \\
\hline Importância da instância regional & $\begin{array}{c}\text { 9,16/ } \\
\text { Satisfatório }\end{array}$ & $\begin{array}{c}3,25 / \\
\text { Insatisfatório }\end{array}$ & $\begin{array}{c}\text { 7,19 } \\
\text { Satisfatório }\end{array}$ & $\begin{array}{c}5,95 \\
\text { Intermediário }\end{array}$ & $\begin{array}{c}9,55 \\
\text { Satisfatório }\end{array}$ & $\begin{array}{c}5,50 / \\
\text { Intermediário }\end{array}$ \\
\hline Política & $\begin{array}{c}\text { 8,15/ } \\
\text { Satisfatório }\end{array}$ & $\begin{array}{c}4,33 / \\
\text { Insatisfatório }\end{array}$ & $\begin{array}{c}\text { 7,05/ } \\
\text { Satisfatório }\end{array}$ & $\begin{array}{c}\text { 6,40/ } \\
\text { Intermediário }\end{array}$ & $\begin{array}{c}\text { 8,50/ } \\
\text { Satisfatório }\end{array}$ & $\begin{array}{c}5,69 \\
\text { Intermediário }\end{array}$ \\
\hline Mobilização de apoios para ações & $\begin{array}{c}7,84 / \\
\text { Satisfatório }\end{array}$ & $\begin{array}{c}5,37 \\
\text { Intermediário }\end{array}$ & $\begin{array}{c}6,46 \\
\text { Intermediário }\end{array}$ & $\begin{array}{c}6,66 \\
\text { Intermediário }\end{array}$ & $\begin{array}{c}\text { 7,24/ } \\
\text { Satisfatório }\end{array}$ & $\begin{array}{c}\text { 7,36/ } \\
\text { Satisfatório }\end{array}$ \\
\hline Suficiência financeira & $\begin{array}{c}5,01 / \\
\text { Intermediário }\end{array}$ & $\begin{array}{c}2,70 / \\
\text { Insatisfatório }\end{array}$ & $\begin{array}{c}2,88 / \\
\text { Insatisfatório }\end{array}$ & $\begin{array}{c}3,20 / \\
\text { Insatisfatório }\end{array}$ & $\begin{array}{c}\text { 3,85/ } \\
\text { Insatisfatório }\end{array}$ & $\begin{array}{c}2,85 / \\
\text { Insatisfatório }\end{array}$ \\
\hline Suficiência de recursos humanos & $\begin{array}{c}4,66 / \\
\text { Insatisfatório }\end{array}$ & $\begin{array}{c}\text { 3,53/ } \\
\text { Insatisfatório }\end{array}$ & $\begin{array}{c}3,25 / \\
\text { Insatisfatório }\end{array}$ & $\begin{array}{c}2,80 / \\
\text { Insatisfatório }\end{array}$ & $\begin{array}{c}4,38 / \\
\text { Insatisfatório }\end{array}$ & $\begin{array}{c}5,50 / \\
\text { Intermediário }\end{array}$ \\
\hline Suficiência de recursos físicos & $\begin{array}{c}8,50 / \\
\text { Satisfatório }\end{array}$ & $\begin{array}{c}5,72 / \\
\text { Intermediário }\end{array}$ & $\begin{array}{c}\text { 6,76/ } \\
\text { Intermediário }\end{array}$ & $\begin{array}{c}\text { 7,34/ } \\
\text { Satisfatório }\end{array}$ & $\begin{array}{c}\text { 7,32/ } \\
\text { Satisfatório }\end{array}$ & $\begin{array}{c}\text { 6,31/ } \\
\text { Intermediário }\end{array}$ \\
\hline Estrutura & $\begin{array}{c}6,50 / \\
\text { Intermediário }\end{array}$ & $\begin{array}{c}\text { 4,33/ } \\
\text { Insatisfatório }\end{array}$ & $\begin{array}{c}4,84 / \\
\text { Insatisfatório }\end{array}$ & $\begin{array}{c}5,00 / \\
\text { Intermediário }\end{array}$ & $\begin{array}{c}5,69 / \\
\text { Intermediário }\end{array}$ & $\begin{array}{c}5,50 / \\
\text { Intermediário }\end{array}$ \\
\hline Planejamento e gestão & $\begin{array}{c}\text { 8,78/ } \\
\text { Satisfatório }\end{array}$ & $\begin{array}{c}\text { 6,84/ } \\
\text { Intermediário }\end{array}$ & $\begin{array}{c}\text { 8,36/ } \\
\text { Satisfatório }\end{array}$ & $\begin{array}{c}7,45 / \\
\text { Satisfatório }\end{array}$ & $\begin{array}{c}8,421 \\
\text { Satisfatório }\end{array}$ & $\begin{array}{c}\text { 7,14/ } \\
\text { Satisfatório }\end{array}$ \\
\hline Integração & $\begin{array}{c}\text { 8,35/ } \\
\text { Satisfatório }\end{array}$ & $\begin{array}{c}5,09 / \\
\text { Intermediário }\end{array}$ & $\begin{array}{c}\text { 8,86/ } \\
\text { Satisfatório }\end{array}$ & $\begin{array}{c}\text { 7,84/ } \\
\text { Satisfatório }\end{array}$ & $\begin{array}{c}5,88 / \\
\text { Intermediário }\end{array}$ & $\begin{array}{c}\text { 9,36/ } \\
\text { Satisfatório }\end{array}$ \\
\hline $\begin{array}{l}\text { Controle de determinantes, riscos e danos } \\
\text { à saúde }\end{array}$ & $\begin{array}{c}\text { 6,92/ } \\
\text { Intermediário }\end{array}$ & $\begin{array}{c}4,87 / \\
\text { Insatisfatório }\end{array}$ & $\begin{array}{c}\text { 7,40/ } \\
\text { Satisfatório }\end{array}$ & $\begin{array}{c}\text { 6,41/ } \\
\text { Intermediário }\end{array}$ & $\begin{array}{c}5,64 / \\
\text { Intermediário }\end{array}$ & $\begin{array}{c}\text { 6,82/ } \\
\text { Intermediário }\end{array}$ \\
\hline Organização & $\begin{array}{c}8,02 / \\
\text { Satisfatório }\end{array}$ & $\begin{array}{c}5,60 / \\
\text { Insatisfatório }\end{array}$ & $\begin{array}{c}8,20 / \\
\text { Satisfatório }\end{array}$ & $\begin{array}{c}\text { 7,23/ } \\
\text { Satisfatório }\end{array}$ & $\begin{array}{c}\text { 6,65/ } \\
\text { Intermediário }\end{array}$ & $\begin{array}{c}\text { 7,77/ } \\
\text { Satisfatório }\end{array}$ \\
\hline Desempenho da região & $\begin{array}{c}7,56 / \\
\text { Satisfatório }\end{array}$ & $\begin{array}{c}4,75 / \\
\text { Insatisfatório }\end{array}$ & $\begin{array}{c}6,70 \\
\text { Intermediário }\end{array}$ & $\begin{array}{c}\text { 6,21/ } \\
\text { Intermediário }\end{array}$ & $\begin{array}{c}\text { 6,95/ } \\
\text { Intermediário }\end{array}$ & $\begin{array}{c}\text { 6,32/ } \\
\text { Intermediário }\end{array}$ \\
\hline
\end{tabular}


Na dimensão Política, as RS I, RS III e RS V alcançaram desempenho satisfatório, reconhecendo a importância da instância regional na tomada de decisão e na operacionalização das ações de vigilância em saúde, enquanto a RS II foi a única com desempenho insatisfatório. No atributo "protagonismo da região”, as RS I e RS V apresentaram desempenho satisfatório, e a RS II, insatisfatório, destacando-se os órgãos/instituições que influenciaram este resultado: "organizações da sociedade civil”, "instituições acadêmicas” e Conselho de Secretários Municipais de Saúde (COSEMS). O único órgão/instituição que atingiu resultado satisfatório em todas as regiões no atributo "protagonismo da região" foi a Secretaria Estadual de Saúde (SES) - nível central (Tabela 4).

A dimensão Estrutura atingiu desempenho intermediário (RS I, RS IV, RSV e RSVI) ou insatisfatório (RS II e RS III), com os atributos "suficiência financeira" e "suficiência de recursos humanos" obtendo os piores resultados. Com relação à “suficiência financeira”, ressalta-se nas RS I, RS II, RS III e RS IV a participação da esfera federal no custeio da vigilância em saúde, em detrimento das estadual e municipal. Em todas as regiões, o percentual de execução dos recursos do Piso Fixo de Vigilância e Promoção da Saúde e o investimento na vigilância em saúde nos últimos três anos registraram resultado negativo (Tabela 4).

\section{Tabela 4}

Índice de desempenho da regionalização da vigilância em saúde, segundo as dimensões Política e Estrutura, atributos, indicadores e variáveis, por Região de Saúde. Brasil, 2017.

\begin{tabular}{|c|c|c|c|c|c|c|}
\hline \multirow[t]{2}{*}{ Dimensão/Atributo/Indicador/Variáveis } & \multicolumn{6}{|c|}{ Escore médio da Região de Saúde * } \\
\hline & $\mathbf{I}$ & II & III & IV & $\mathbf{V}$ & VI \\
\hline \multicolumn{7}{|l|}{ Política } \\
\hline \multicolumn{7}{|l|}{ Protagonismo da Região de Saúde } \\
\hline Importância dos órgãos para a tomada de decisões & 7,15 & 5,41 & 6,91 & 6,85 & 7,45 & 5,88 \\
\hline Secretaria Estadual de Saúde (nível central) & 7,48 & 8,88 & 7,75 & 9,10 & 8,20 & 7,30 \\
\hline Estrutura regional da Secretaria Estadual de Saúde & 7,48 & 7,75 & 7,75 & 9,10 & 6,85 & 8,20 \\
\hline Organizações da sociedade civil & 6,06 & 2,69 & 6,06 & 3,70 & 6,40 & 4,60 \\
\hline Instituições acadêmicas & 5,50 & 3,25 & 5,50 & 3,70 & 7,30 & 4,15 \\
\hline COSEMS & 8,06 & 5,50 & 7,75 & 7,30 & 7,30 & 5,05 \\
\hline $\mathrm{CIR}$ & 8,31 & 4,38 & 6,63 & 8,20 & 8,65 & 5,95 \\
\hline Desempenho do atributo & 7,15 & 5,41 & 6,91 & 6,85 & 7,45 & 5,88 \\
\hline \multicolumn{7}{|l|}{ Importância da instância regional } \\
\hline Frequência nas reuniões da CIR de temas da vigilância em saúde & 9,16 & 3,25 & 7,19 & 5,95 & 9,55 & 5,50 \\
\hline Desempenho do atributo & 9,16 & 3,25 & 7,19 & 5,95 & 9,55 & 5,50 \\
\hline Resultado do índice sintético - Política & 8,15 & 4,33 & 7,05 & 6,40 & 8,50 & 5,69 \\
\hline \multicolumn{7}{|l|}{ Estrutura } \\
\hline \multicolumn{7}{|l|}{ Mobilização de apoio para as ações } \\
\hline Frequência na mobilização de apoios necessários para & 7,84 & 5,37 & 6,46 & 6,66 & 7,24 & 7,36 \\
\hline Vigilância, prevenção e controle das doenças transmissíveis & 8,65 & 8,43 & 7,75 & 7,75 & 8,65 & 9,55 \\
\hline Vigilância e prevenção de agravos não transmissíveis & 7,30 & 4,38 & 5,50 & 5,05 & 7,75 & 6,85 \\
\hline Vigilância de população expostas a riscos ambientais em saúde & 7,52 & 3,25 & 4,94 & 7,75 & 7,30 & 4,60 \\
\hline Vigilância de saúde do trabalhador & 7,30 & 2,13 & 6,63 & 6,40 & 6,40 & 7,30 \\
\hline Promoção em saúde e hábitos de vida saudáveis & 6,85 & 6,18 & 3,81 & 3,70 & 5,50 & 6,40 \\
\hline Vigilância sanitária & 8,42 & 5,50 & 8,31 & 8,20 & 6,40 & 7,30 \\
\hline Gestão dos sistemas de informação de vigilância em saúde & 8,87 & 7,75 & 8,31 & 7,75 & 8,65 & 9,55 \\
\hline
\end{tabular}

(continua) 


\begin{tabular}{|c|c|c|c|c|c|c|}
\hline \multirow[t]{2}{*}{ Dimensão/Atributo/Indicador/Variáveis } & \multicolumn{6}{|c|}{ Escore médio da Região de Saúde * } \\
\hline & $\mathbf{I}$ & II & III & IV & $\mathbf{v}$ & VI \\
\hline \multicolumn{7}{|l|}{ Estrutura } \\
\hline Desempenho do atributo & 7,84 & 5,37 & 6,46 & 6,66 & 7,24 & 7,36 \\
\hline \multicolumn{7}{|l|}{ Suficiência financeira } \\
\hline Custeio da vigilância em saúde & 6,29 & 6,10 & 5,50 & 6,70 & 5,05 & 5,65 \\
\hline Federal & 7,07 & 7,30 & 8,88 & 7,30 & 5,95 & 5,50 \\
\hline Estadual & 4,95 & 5,50 & 4,94 & 6,40 & 3,70 & 5,05 \\
\hline Municipal & 6,85 & 5,50 & 2,69 & 6,40 & 5,50 & 6,40 \\
\hline $\begin{array}{l}\text { \% de execução dos recursos do Piso Fixo e Variável da Vigilância e Promoção da } \\
\text { Saúde (últimos três anos) }\end{array}$ & 3,25 & 1,00 & 2,13 & 1,90 & 1,90 & 1,90 \\
\hline Entre $75 \%$ e $100 \%$ & 3,25 & 1,00 & 3,25 & 2,80 & 1,00 & 2,80 \\
\hline $100 \%$ & 3,25 & 1,00 & 1,00 & 1,00 & 2,80 & 1,00 \\
\hline $\begin{array}{l}\text { Aumento no investimento para vigilância em saúde na região, nos } \\
\text { últimos três anos }\end{array}$ & 5,50 & 1,00 & 1,00 & 1,00 & 4,60 & 1,00 \\
\hline $\begin{array}{l}\text { Desempenho do atributo } \\
\text { Suficiência de recursos humanos }\end{array}$ & 5,01 & 2,70 & 2,88 & 3,20 & 3,85 & 2,85 \\
\hline Suficiência de recursos humanos para vigilância em saúde & 4,66 & 3,53 & 3,25 & 2,80 & 4,38 & 5,50 \\
\hline Médicos epidemiologistas & 3,25 & 1,00 & 1,00 & 1,00 & 1,00 & 2,80 \\
\hline Sanitaristas & 3,25 & 5,50 & 1,00 & 1,00 & 1,00 & 2,80 \\
\hline Inspetores sanitários & 3,25 & 3,25 & 1,00 & 2,80 & 2,80 & 2,80 \\
\hline Codificadores de causa básica & 5,50 & 1,00 & 10,00 & 4,60 & 6,40 & 8,20 \\
\hline Agentes de endemias para as ações de campo & 3,25 & 1,00 & 3,25 & 4,60 & 6,40 & 4,60 \\
\hline Digitadores para os sistemas de informação da vigilância em saúde & 7,75 & 7,75 & 3,25 & 4,60 & 4,60 & 8,20 \\
\hline Outros profissionais de nível superior & 5,50 & 3,25 & 1,00 & 2,80 & 6,40 & 6,40 \\
\hline Outros profissionais de nível médio & 5,50 & 5,50 & 5,50 & 1,00 & 6,40 & 8,20 \\
\hline Desempenho do atributo & 4,66 & 3,53 & 3,25 & 2,80 & 4,38 & 5,50 \\
\hline \multicolumn{7}{|l|}{ Suficiência de recursos físicos } \\
\hline Suficiência da estrutura da vigilância em saúde & 8,88 & 6,25 & 4,00 & 5,20 & 5,80 & 4,60 \\
\hline Estrutura física (salas, mesas, cadeiras etc.) & 8,88 & 3,25 & 1,00 & 6,40 & 4,60 & 2,80 \\
\hline Veículos exclusivos & 5,50 & 5,50 & 1,00 & 2,80 & 2,80 & 2,80 \\
\hline Computadores conectados à Internet, impressoras e telefones & 8,88 & 3,25 & 3,25 & 4,60 & 6,40 & 4,60 \\
\hline Material gráfico para divulgação das ações, programas e campanhas & 10,00 & 5,50 & 3,25 & 6,40 & 4,60 & 4,60 \\
\hline Impressos e normas & 10,00 & 10,00 & 7,75 & 6,40 & 8,20 & 6,40 \\
\hline Material de expediente & 10,00 & 10,00 & 7,75 & 4,60 & 8,20 & 6,40 \\
\hline Frequência de procedimentos relativos à vigilância da tuberculose & 8,65 & 4,69 & 8,09 & 7,17 & 7,17 & 7,17 \\
\hline Teste rápido para detecção de HIV nos casos de tuberculose & 8,87 & 3,93 & 4,38 & 3,93 & 3,93 & 3,93 \\
\hline Quimioprofilaxia nos comunicantes, quando necessário & 7,75 & 4,38 & 8,88 & 5,95 & 5,95 & 5,95 \\
\hline Exames de baciloscopia em todos os casos suspeitos & 8,87 & 5,05 & 7,75 & 9,55 & 9,55 & 9,55 \\
\hline Antirretrovirais em casos de coinfecção tuberculose/HIV & 8,87 & 5,05 & 9,44 & 6,40 & 6,40 & 6,40 \\
\hline Esquema básico para todos os casos confirmados de tuberculose & 8,87 & 5,05 & 10,00 & 10,00 & 10,00 & 10,00 \\
\hline Frequência de procedimentos relativos à vigilância e controle de dengue & 7,97 & 6,22 & 8,20 & 9,64 & 8,99 & 7,15 \\
\hline Disponibilidade de sorologia no nível regional & 8,87 & 6,63 & 7,75 & 10,00 & 7,75 & 6,25 \\
\hline Disponibilidade de insumos para o controle vetorial & 7,52 & 7,30 & 9,44 & 9,55 & 8,88 & 8,50 \\
\hline Disponibilidade de medicamentos básicos para dengue & 7,75 & 6,18 & 8,88 & 9,10 & 9,44 & 9,25 \\
\hline Disponibilidade de impressos & 8,20 & 6,63 & 8,88 & 10,00 & 10,00 & 5,50 \\
\hline $\begin{array}{l}\text { Aquisição de equipamento de proteção individual para aplicação de inseticidas } \\
\text { e larvicidas }\end{array}$ & 7,52 & 4,38 & 6,06 & 9,55 & 8,88 & 6,25 \\
\hline Desempenho do atributo & 8,50 & 5,72 & 6,76 & 7,34 & 7,32 & 6,31 \\
\hline Resultado do índice sintético - Estrutura & 6,50 & 4,33 & 4,84 & 5,00 & 5,69 & 5,50 \\
\hline
\end{tabular}

CIR: Comissão Intergestores Regional; COSEMS: Conselho de Secretários Municipais de Saúde.

Nota: classificação EM: insatisfatório: $\leq 4,99$; intermediário: entre 5,00 e 6,99; e satisfatório: $\geq 7,00$.

* Região de Saúde I: V Diretoria Regional de Saúde de São Paulo; Região de Saúde II: Região do Entorno de Manaus e Alto do Rio Negro; Região de Saúde III: Região da Baixada Cuiabana; Região de Saúde IV: Região de Carbonífera/Costa Doce; Região de Saúde V: VIII Região de Saúde de Pernambuco; e Região de Saúde VI: Núcleo Regional de Saúde Norte da Bahia. 
Quanto à "suficiência de recursos humanos", os itens "médicos epidemiologistas", "sanitaristas" e "inspetores sanitários” foram insatisfatórios em todas as regiões, já "digitadores para os sistemas de informação da vigilância em saúde" foram satisfatórios nas RS I, RS II e RS VI; e "codificadores de causa básica” nas RS III e RS VI. Ao analisar a "suficiência de recursos físicos”, os itens referentes aos agravos traçadores tuberculose e dengue apresentaram resultado satisfatório em todas as regiões, com exceção da RS II (Tabela 4).

Com relação à dimensão Organização, percebeu-se a importância do "planejamento e gestão" em todas as regiões, exceto na RS II, que alcançou resultado intermediário. O atributo "integração" apresentou desempenho satisfatório nas RS I, RS III, RS IV e RS VI; e o "controle de determinantes, riscos e danos à saúde”, apenas na RS III (Tabela 5).

No que se refere às práticas e processos de trabalho da vigilância em saúde, a RS II atingiu resultado satisfatório apenas nos itens "vigilância da situação de saúde da população" e "vigilância, prevenção e controle das doenças transmissíveis”. Observou-se desempenho insatisfatório da RS V relacionado com a "existência de integração intrassetorial da vigilância em saúde" e da RS II no que diz respeito à "existência de integração entre a vigilância em saúde e os demais serviços de saúde" (Tabela 5).

Quanto à "frequência no envio de amostra laboratorial de exames de interesse para a vigilância em saúde para análise em outra região”, as RS I, RS II, RS IV, RS V e RS VI registraram resultado insatisfatório, e apenas a RS II no que se refere à "frequência de encerramento por critério laboratorial dos casos de doenças de notificação compulsória”. Ressalta-se o resultado insatisfatório das RS II e RS V em relação à "existência de acompanhamento dos casos de tuberculose resistente e tuberculose multidroga resistente na região” (Tabela 5).

\section{Discussão}

Os resultados deste estudo mostram que, em geral, quanto maior o nível de desenvolvimento socioeconômico e da oferta de serviços, melhor o desempenho da regionalização da vigilância em saúde, e que as dimensões Organização e Política alcançam melhores escores e a Estrutura apresenta maior debilidade.

A opção por avaliar o desempenho da regionalização da vigilância em saúde na amplitude do seu constructo em detrimento das especificidades de cada uma das vigilâncias (epidemiológica, sanitária, saúde do trabalhador, ambiental) que a compõem permitiu uma análise global do desempenho, mas fragilizou a avaliação em profundidade de cada uma delas. Adicionalmente, a seleção dos municípios nas Regiões de Saúde com base no critério de conveniência, exceto o município-sede, ao priorizar aqueles com maior adesão à pesquisa e que receberam os pesquisadores nas datas propostas, pode ter sobrevalorizado o desempenho da vigilância em saúde na região. A aplicação do questionário tanto aos gestores quanto aos profissionais da vigilância em saúde buscou, ao confrontar fontes, ampliar a confiabilidade das informações.

A Região de Saúde caracterizada pelo alto desenvolvimento socioeconômico e pela alta oferta de serviços, RS I (grupo 5), apresentou desempenho satisfatório em duas dimensões (Política e Organização), já as de níveis socioeconômicos e de oferta de serviços mais baixos, RS IV, RS V e RS VI, integrantes dos grupos 3, 2 e 1, respectivamente, apenas em uma dimensão. A exceção foi a de médio/alto desenvolvimento socioeconômico e baixa oferta de serviços, RS II (grupo 4), que apresentou resultado insatisfatório em todas as dimensões.

O desempenho insatisfatório da RS II, apesar da tipologia, decorre possivelmente do contexto local, estado de dimensão continental e municípios extensos, com meios de transporte precários, muitas vezes por via fluvial, o que dificulta a integração das ações e serviços em âmbito regional 31 . Adicionalmente, a Fundação de Vigilância em Saúde, órgão vinculado à Secretaria de Saúde do Amazonas, atua, por vezes, cartorialmente, protagonizando ações, a exemplo da disponibilização de agentes de endemias na quase totalidade dos municípios, inclusive na capital. Há evidências de que a Região Amazônica requer intervenções complexas e que estas são particularmente sensíveis aos contextos organizacionais em que se inserem, com repercussões sobre o seu nível de implantação e desempenho 31 
Tabela 5

Índice de desempenho da regionalização da vigilância em saúde, segundo a dimensão Organização, atributos, indicadores e variáveis, por Região de Saúde. Brasil, 2017.

\begin{tabular}{|c|c|c|c|c|c|c|}
\hline \multirow[t]{2}{*}{ Dimensão/Atributo/Indicador/Variáveis } & \multicolumn{6}{|c|}{ Escore médio da Região de Saúde * } \\
\hline & I & II & III & IV & v & VI \\
\hline \multicolumn{7}{|l|}{ Organização } \\
\hline \multicolumn{7}{|l|}{ Planejamento e gestão } \\
\hline Utilização de critérios para o planejamento da vigilância em saúde na região & 8,43 & 6,85 & 9,10 & 6,40 & 8,56 & 7,12 \\
\hline Critérios epidemiológicos & 10,00 & 10,00 & 10,00 & 8,20 & 10,00 & 8,20 \\
\hline Caracterização da população (perfil socioeconômico e demográfico) & 10,00 & 7,75 & 10,00 & 8,20 & 10,00 & 8,20 \\
\hline Caracterização das condições ambientais (água, coleta de lixo e dejetos) & 7,75 & 7,75 & 10,00 & 6,40 & 10,00 & 8,20 \\
\hline Dimensão e distribuição da rede de serviços de assistência à saúde & 10,00 & 5,50 & 10,00 & 6,40 & 10,00 & 8,20 \\
\hline $\begin{array}{l}\text { Resultados da avaliação das capacidades de respostas do Regulamento } \\
\text { Sanitário Internacional }\end{array}$ & 4,38 & 3,25 & 5,50 & 2,80 & 2,80 & 2,80 \\
\hline $\begin{array}{l}\text { Existência de definição clara das atribuições/funções dos gestores da } \\
\text { vigilância em saúde }\end{array}$ & 8,50 & 7,75 & 10,00 & 9,40 & 6,40 & 6,40 \\
\hline Federal & 8,88 & 7,75 & 10,00 & 8,20 & 8,20 & 6,40 \\
\hline Estadual & 7,75 & 7,75 & 10,00 & 10,00 & 10,00 & 6,40 \\
\hline Municipal & 8,88 & 7,75 & 10,00 & 10,00 & 1,00 & 6,40 \\
\hline Garantia da gestão da vigilância em saúde na região para: & 8,65 & 5,95 & 5,95 & 6,04 & 8,20 & 6,04 \\
\hline Fortalecimento da capacidade institucional & 7,75 & 5,50 & 3,25 & 8,20 & 8,20 & 2,80 \\
\hline Articulação intra e intersetorial & 8,88 & 7,75 & 7,75 & 6,40 & 8,20 & 8,20 \\
\hline Produção de informação e comunicação & 8,88 & 7,75 & 5,50 & 6,40 & 8,20 & 4,60 \\
\hline Análise da situação de saúde & 10,00 & 7,75 & 10,00 & 6,40 & 10,00 & 8,20 \\
\hline Aprimoramento da gestão financeira (alocação e execução) & 7,75 & 1,00 & 3,25 & 2,80 & 6,40 & 6,40 \\
\hline Contribuição da gestão da vigilância em saúde na região & 9,44 & 8,13 & 7,38 & 6,70 & 9,70 & 8,20 \\
\hline Aprimoramento institucional & 8,88 & 7,75 & 5,50 & 6,40 & 10,00 & 6,40 \\
\hline Fortalecimento da gestão regional & 10,00 & 7,75 & 3,25 & 4,60 & 10,00 & 8,20 \\
\hline Fortalecimento das ações intersetoriais & 8,88 & 7,75 & 7,75 & 4,60 & 10,00 & 8,20 \\
\hline Prevenção e controle de doenças e agravos & 10,00 & 7,75 & 10,00 & 10,00 & 10,00 & 10,00 \\
\hline Promoção da saúde & 10,00 & 10,00 & 7,75 & 10,00 & 10,00 & 8,20 \\
\hline Integração dos serviços de vigilância em saúde com outros setores na região & 8,88 & 7,75 & 10,00 & 4,60 & 8,20 & 8,20 \\
\hline $\begin{array}{l}\text { Existência de práticas e processos de trabalho da vigilância em saúde } \\
\text { voltados para }\end{array}$ & 8,88 & 5,50 & 9,36 & 8,71 & 9,23 & 7,94 \\
\hline Vigilância da situação de saúde da população & 8,88 & 7,75 & 7,75 & 6,40 & 8,20 & 6,40 \\
\hline Detecção oportuna e adoção de medidas para a resposta às emergências & 8,88 & 5,50 & 10,00 & 8,20 & 8,20 & 6,40 \\
\hline Vigilância, prevenção e controle das doenças transmissíveis & 8,88 & 7,75 & 10,00 & 10,00 & 10,00 & 10,00 \\
\hline Vigilância das doenças crônicas não transmissíveis, dos acidentes e violências & 7,75 & 3,25 & 10,00 & 10,00 & 10,00 & 8,20 \\
\hline Vigilância de populações expostas a riscos ambientais em saúde & 8,88 & 5,50 & 7,75 & 6,40 & 10,00 & 6,40 \\
\hline Vigilância da saúde do trabalhador & 10,00 & 3,25 & 10,00 & 10,00 & 10,00 & 8,20 \\
\hline Vigilância sanitária & 8,88 & 5,50 & 10,00 & 10,00 & 8,20 & 10,00 \\
\hline
\end{tabular}

(continua) 


\begin{tabular}{|c|c|c|c|c|c|c|}
\hline & \multirow[b]{2}{*}{ I } & \multirow[b]{2}{*}{ II } & & & & \\
\hline & & & III & IV & V & VI \\
\hline \multicolumn{7}{|l|}{ Organização } \\
\hline Desempenho do atributo & 8,78 & 6,84 & 8,36 & 7,45 & 8,42 & 7,14 \\
\hline \multicolumn{7}{|l|}{ Integração } \\
\hline Existência de integração intrassetorial da vigilância em saúde & 7,75 & 5,50 & 7,75 & 8,20 & 2,80 & 10,00 \\
\hline $\begin{array}{l}\text { Existência de integração entre a vigilância em saúde e os demais serviços de } \\
\text { saúde }\end{array}$ & 10,00 & 3,25 & 10,00 & 8,20 & 6,40 & 10,00 \\
\hline $\begin{array}{l}\text { Importância de itens para a promoção da integração entre a vigilância em saúde } \\
\text { e atenção primária à saúde }\end{array}$ & 7,31 & 6,53 & 8,83 & 7,11 & 8,44 & 8,07 \\
\hline Organização conjunta do processo de trabalho & 8,26 & 8,88 & 9,44 & 7,30 & 8,88 & 8,71 \\
\hline Mecanismos de planejamento e programação conjuntos & 7,93 & 8,88 & 9,44 & 8,20 & 8,65 & 9,04 \\
\hline Práticas conjuntas de educação permanente em saúde & 8,08 & 7,75 & 9,44 & 6,85 & 8,65 & 8,71 \\
\hline Mapeamento e avaliação conjuntos & 8,08 & 7,75 & 8,88 & 6,85 & 7,98 & 9,04 \\
\hline Participação em comitês conjuntos (p. ex: investigação de óbitos) & 7,41 & 6,63 & 8,31 & 7,30 & 7,98 & 8,71 \\
\hline Discussão conjunta de casos clínicos e eventos sentinelas & 6,89 & 6,06 & 7,75 & 7,30 & 9,10 & 8,07 \\
\hline Realização de visitas domiciliares e/ou atendimentos compartilhados & 6,89 & 3,81 & 9,44 & 7,75 & 8,88 & 7,43 \\
\hline Realização de ações coletivas intra e intersetoriais no território & 7,57 & 6,63 & 8,88 & 6,85 & 8,43 & 8,07 \\
\hline Ações conjuntas entre os ACS e os ACE & 7,57 & 6,63 & 9,44 & 7,30 & 8,43 & 9,04 \\
\hline Vigilância de eventos adversos e farmacovigilância & 6,89 & 4,94 & 8,31 & 6,85 & 8,20 & 7,11 \\
\hline Vigilância alimentar e nutricional & 6,44 & 5,50 & 8,31 & 7,75 & 8,20 & 6,14 \\
\hline Ações conjuntas de promoção de atividades físicas & 5,68 & 4,94 & 8,31 & 5,05 & 7,98 & 6,79 \\
\hline Desempenho do atributo & 8,35 & 5,09 & 8,86 & 7,84 & 5,88 & 9,36 \\
\hline \multicolumn{7}{|l|}{ Controle de determinantes, riscos e danos à saúde } \\
\hline Frequência no envio de amostras laboratoriais para análise em outra região & 2,69 & 4,94 & 6,06 & 2,80 & 2,80 & 2,35 \\
\hline $\begin{array}{l}\text { Frequência de encerramento por critério laboratorial dos casos de doenças de } \\
\text { notificação compulsória }\end{array}$ & 7,19 & 3,25 & 7,19 & 7,30 & 8,65 & 7,30 \\
\hline Frequência na realização de ações referentes aos casos de tuberculose & 6,55 & 5,07 & 7,05 & 6,48 & 7,75 & 7,56 \\
\hline Busca ativa de sintomáticos respiratórios no território & 5,73 & 5,05 & 6,63 & 5,05 & 6,25 & 5,50 \\
\hline Identificação de sinais e sintomas de tuberculos & 6,63 & 5,50 & 7,19 & 6,85 & 8,50 & 8,88 \\
\hline Notificação dos casos diagnosticados & 7,75 & 6,63 & 9,44 & 9,10 & 10,00 & 10,00 \\
\hline Tratamento dos casos de tuberculose de acordo com o protocolo de & 7,30 & 5,50 & 8,31 & 6,40 & 9,25 & 7,75 \\
\hline \multicolumn{7}{|l|}{ Tratamento Diretamente Observado } \\
\hline Aconselhamento pré e pós-teste & 7,53 & 5,05 & 6,63 & 7,75 & 8,50 & 6,63 \\
\hline Busca ativa de faltosos ao tratamento & 7,53 & 3,25 & 7,75 & 7,75 & 5,50 & 8,88 \\
\hline Encerramento dos casos no livro de registro de pacientes & 6,40 & 4,38 & 8,31 & 9,10 & 7,00 & 8,88 \\
\hline $\begin{array}{l}\text { Acompanhamento do tratamento com registro no boletim de } \\
\text { acompanhamento }\end{array}$ & 6,40 & 6,18 & 5,50 & 5,05 & 8,50 & 9,44 \\
\hline $\begin{array}{l}\text { Monitoramento de encerramento dos casos tratados com envio da } \\
\text { informação regular e oportuna para a vigilância em saúde }\end{array}$ & 6,40 & 4,38 & 7,19 & 7,75 & 10,00 & 8,88 \\
\hline Realização de atividade de educação em saúde sobre tuberculose & 6,40 & 6,18 & 6,63 & 4,15 & 7,00 & 5,50 \\
\hline Divulgação de informações sobre a situação epidemiológica da tuberculose & 5,50 & 4,38 & 6,06 & 4,60 & 7,00 & 5,50 \\
\hline $\begin{array}{l}\text { Estímulo à organização e à participação da sociedade civil no controle da } \\
\text { tuberculose }\end{array}$ & 5,05 & 4,38 & 4,94 & 4,15 & 5,50 & 4,94 \\
\hline
\end{tabular}

(continua) 


\begin{tabular}{|c|c|c|c|c|c|c|}
\hline \multirow[t]{2}{*}{ Dimensão/Atributo/Indicador/Variáveis } & \multicolumn{6}{|c|}{ Escore médio da Região de Saúde * } \\
\hline & I & II & III & IV & v & VI \\
\hline \multicolumn{7}{|l|}{ Organização } \\
\hline $\begin{array}{l}\text { Frequência com que os dados relacionados com a tuberculose são consolidados } \\
\text { e analisados }\end{array}$ & 8,87 & 5,50 & 5,50 & 6,40 & 5,50 & 7,30 \\
\hline $\begin{array}{l}\text { Acompanhamento dos casos de tuberculose resistente e multidroga resistente } \\
\text { na região }\end{array}$ & 7,75 & 3,25 & 10,00 & 6,40 & 1,00 & 7,80 \\
\hline Realização de ações de controle do dengue na região & 8,45 & 7,19 & 8,59 & 9,10 & 8,13 & 8,59 \\
\hline Notificação dos casos de dengue pelas UBS/USF & 8,88 & 10,00 & 10,00 & 10,00 & 10,00 & 10,00 \\
\hline Investigação dos casos de dengue pelas UBS/USF & 6,63 & 3,25 & 5,50 & 8,20 & 7,00 & 10,00 \\
\hline Análise de consistência, completude e fluxo de dados do SINAN & 8,88 & 5,50 & 10,00 & 10,00 & 10,00 & 10,00 \\
\hline Elaboração de mapas das situações epidemiológicas e entomológicas & 7,75 & 7,75 & 10,00 & 10,00 & 10,00 & 3,25 \\
\hline Unificação da base geográfica de trabalho (vigilância em saúde e PACS/PSF) & 8,88 & 5,50 & 3,25 & 10,00 & 4,00 & 7,75 \\
\hline Divulgação do protocolo de assistência aos pacientes com dengue & 8,88 & 7,75 & 10,00 & 8,20 & 7,00 & 10,00 \\
\hline Capacitação de profissionais de saúde & 8,88 & 7,75 & 10,00 & 8,20 & 10,00 & 10,00 \\
\hline Realização de atividades de campo integradas entre as vigilâncias & 8,88 & 10,00 & 10,00 & 8,20 & 7,00 & 7,75 \\
\hline Desempenho do atributo & 6,92 & 4,87 & 7,40 & 6,41 & 5,64 & 6,82 \\
\hline Resultado do índice sintético - Organização & 8,02 & 5,60 & 8,20 & 7,23 & 6,65 & 7,77 \\
\hline
\end{tabular}

ACE: agentes de controle de endemias; ACS: agentes comunitários de saúde; PACS: Programa de Agentes Comunitários de Saúde; PSF: Programa Saúde da Família; SINAN: Sistema de Informaç̃̃es de Agravos de Notificação; UBS: unidade básica de saúde; USF: unidade de saúde da família.

Nota: classificação EM: insatisfatório: $\leq 4,99$; intermediário: entre 5,00 e 6,99; e satisfatório: $\geq 7,00$.

* Região de Saúde I: V Diretoria Regional de Saúde de São Paulo; Região de Saúde II: Região do Entorno de Manaus e Alto do Rio Negro; Região de Saúde III: Região da Baixada Cuiabana; Região de Saúde IV: Região de Carbonífera/Costa Doce; Região de Saúde V: VIII Região de Saúde de Pernambuco; e Região de Saúde VI: Núcleo Regional de Saúde Norte da Bahia.

Os achados deste trabalho corroboram os resultados da pesquisa geral 27, que aponta para um melhor desempenho da dimensão Política ante as demais, e apresenta a Estrutura como a mais precária em todas as regiões. Também apresenta a RS II como tendo o pior desempenho da regionalização e a RS I em uma posição intermediária, sem exercer protagonismo em relação ao processo de regionalização.

Com relação à dimensão Estrutura, a insuficiente disponibilidade de recursos físicos, humanos e financeiros dificulta o avanço da política de regionalização e o alcance de seu macro-objetivo, a constituição de um sistema de saúde universal e integrado, com ações e serviços coordenados. Os resultados da dimensão Estrutura nas seis Regiões de Saúde avaliadas não traduzem as suas diferenças socioeconômicas, tampouco a tipologia adotada neste estudo, sinalizando uma dificuldade estrutural generalizada da vigilância em saúde no território nacional.

Apesar das iniciativas para fortalecer e qualificar a descentralização da vigilância em saúde, especialmente em relação à infraestrutura e à capacidade técnica, a exemplo do Projeto Vigisus $32 \mathrm{e}$ do Programa de Qualificação das Ações de Vigilância em Saúde (PQAVS) 33, estudos 15,16,17 apontam para a necessidade de esforços adicionais para alcançar um melhor desempenho das práticas de vigilância em saúde no nível local. O resultado deste trabalho mostra que falhas estruturais permanecem como impedimento para a regionalização das ações e serviços de vigilância em saúde.

A despeito do progresso nas políticas de saúde no Brasil ao longo dos anos, a gestão dos recursos humanos convive com dificuldades relacionadas com a precariedade dos vínculos, contratações que não consideram as necessidades do setor, baixos salários e falta de planos de cargos e carreira, entre outros aspectos, acarretando a insuficiência de equipes para a execução das ações, as relações temporárias de trabalho e a baixa qualificação técnica em nível local 16,17.

Em contraponto, a Organização da vigilância em saúde tem se consolidado nos últimos anos valendo-se de sua base normativa e legal, por meio de portarias, políticas e planos, no intuito de nortear e qualificar o planejamento e a gestão democrática do sistema de saúde 29 . No entanto, a 
conformação do sistema de saúde brasileiro interfere diretamente na estruturação e no desempenho da vigilância em saúde. O baixo desempenho do atributo "controle de determinantes, riscos e danos à saúde” em relação aos demais da dimensão Organização - principalmente em decorrência da debilidade na execução de ações de controle e da "frequência no envio de amostra laboratorial de exames de interesse para a vigilância em saúde para análise em outra região" - explicitam esta questão.

A adoção de estratégias de controle, baseadas em programas verticalizados nos anos 2000, apresentou pouco impacto no combate de agravos, como tuberculose, dengue, entre outros. Assim, a incorporação dessas ações na atenção básica, sob a responsabilidade municipal, integradas aos demais serviços da rede de atenção, atuando com o usuário em seu contexto de vida, são instrumentos para ampliar a efetividade dos programas 34,35, porém, a expansão de uma atenção básica pouco resolutiva representa uma barreira para a organização de serviços e para a constituição das Regiões de Saúde 36 . Evidências recentes mostraram que a cobertura da Estratégia Saúde da Família aumenta as chances de sucesso do tratamento de algumas doenças em pessoas pobres, a exemplo da tuberculose, apontando o benefício da proteção social nos desfechos de doenças 37 .

Mesmo diante do acompanhamento longitudinal das pessoas ocorrendo na atenção primária, a continuidade do cuidado pode implicar intervenções diagnósticas e tratamentos de média e alta complexidades, frequentemente indisponíveis em municípios com menores contingentes populacionais. A insuficiente atuação das estruturas regionais das SES, tanto no desenho da rede de prestação de serviços quanto no estabelecimento de mecanismos para sua integração, condição imprescindível para o sucesso dos programas de controle dos agravos, constitui desafios para o avanço do processo de regionalização 27 .

Apesar do descompasso da vigilância em relação ao sistema de saúde 38 , uma vez que seus serviços e ações permaneceram centralizados até o século XX e, quando descentralizados, foram direcionados aos municípios, os resultados da dimensão Política no atributo "importância da instância regional" mostraram a Comissão Intergestores Regional (CIR) como espaço para discussão de temas da vigilância em saúde, em três das seis regiões pesquisadas. Entretanto, há evidências de que os fóruns intergovernamentais estão esvaziando porque as decisões sobre as Regiões de Saúde estão centralizadas nos estados, ou nos municípios-sede 27. O fortalecimento das regiões requer pautas estruturadas desses fóruns para discutir sobre a intersetorialidade das políticas públicas e favorecer a construção do território regional 39 . No caso da vigilância em saúde, esses espaços de pactuação e deliberação, em geral, restringem-se às discussões sobre o financiamento de ações, especialmente quanto ao controle de arboviroses 29 .

A efetivação da regionalização pressupõe laços cooperativos interinstitucionais e o compromisso político que transcendam o atendimento a preceitos legais e normativos, com uma visão para a atuação complementar ${ }^{29}$. A delimitação de ações e serviços de ordem geográfica, por vezes, mascara o reconhecimento de que meios sociopolíticos e institucionais são tão ou mais importantes para superar os desafios impostos pelo espaço natural. Para isso, os contextos adversos precisam ser reconhecidos e considerados no planejamento regional.

A regionalização, mais do que um processo de organização das ações e dos serviços de saúde no território, com foco na integralidade da atenção, é uma construção política que favorece o diálogo entre os atores locais e os gestores federados para o reconhecimento e enfrentamento das necessidades de saúde dos territórios específicos 39 . No entanto, estabelecer os limites entre as ações de vigilância em saúde estritamente local e as que necessitam de articulação regional permanece como desafio, em particular, pela ausência de instruções normativas que regulem o compartilhamento de atribuições, de recursos humanos e de materiais na Região de Saúde, bem como o financiamento regional 29.

Ainda com relação ao financiamento, destaca-se a Portaria no 3.992, de 28 de dezembro de 2017 40, que alterou o repasse dos recursos federais do SUS, restringindo-o a dois blocos - custeio e investimento. Não se sabe que impacto essa mudança trará, ao flexibilizar o uso dos recursos federais da saúde nos âmbitos municipal e estadual, e suas implicações na manutenção e na ampliação dos serviços de vigilância em saúde. Igualmente, há dúvidas quanto às repercussões para as instâncias indutoras de políticas estruturantes da vigilância em saúde, especialmente no que concerne à regionalização. $\mathrm{O}$ nível local é importante na organização do sistema de saúde, mas o planejamento da oferta regionalizada precisa ser regulado e financiado pelo sistema de saúde para ser custo efetivo. 
Novos estudos mostrarão impasses, desafios e saídas desse novo ciclo do SUS, mas é certo que suas debilidades são claras e apontam para a necessidade de lideranças institucionais, a serem exercidas pelos estados; políticas públicas mais efetivas; ambientes organizacionais voltados para cooperação e arranjos de participação e controle social, sem os quais a consolidação da regionalização não será viável 41 .

A análise do desempenho da regionalização da vigilância em saúde apresentada retrata a complexidade de contextos de diferentes regiões brasileiras, o que contribui para o entendimento da dinâmica da regionalização da vigilância em saúde no país, explicitando suas concepções e práticas de gestão e suscitando a necessidade de investimentos adicionais para sua estruturação e produção de normativas que balizem o compartilhamento de atribuições, recursos humanos e materiais na Região de Saúde, bem como de fontes de financiamento regional para potencializar as ações.

\section{Colaboradores}

A. C. Albuquerque concebeu e planejou o estudo, levantou e analisou os dados e redigiu o artigo. E. A. P. Cesse participou da concepção do estudo e colaborou na redação, análise e revisão crítica do artigo. E. Felisberto, I. C. Samico e P. G. Frias participaram da concepção e planejamento do estudo e colaboraram na redação, análise e revisão crítica do artigo.

\section{Informações adicionais}

ORCID: Ana Coelho de Albuquerque (0000-00016305-4127); Eduarda Ângela Pessoa Cesse (00000001-5745-3981); Eronildo Felisberto (0000-00022316-2251); Isabella Chagas Samico (0000-00028338-7946); Paulo Germano de Frias (0000-00034497-8898).

\section{Agradecimentos}

Ao Ministério da Ciência, Tecnologia e Inovação e ao Ministério da Saúde (chamada MCTI/CNPq/CTSaúde/MS/SCTIE/Decit no 41/2013), bem como à Coordenação de Aperfeiçoamento de Pessoal de Nível Superior (Capes), pelo apoio financeiro.

\section{Referências}

1. Paim JS, Travassos C, Almeida C, Bahia L, Macinko J. The Brazilian Health System: history, advances, and challenges. Lancet 2011; 377:1778-97.

2. Viana ALd'A, Machado CV. Descentralização e coordenação federativa: a experiência brasileira na saúde. Ciênc Saúde Colet 2009; 14:80717.

3. Lima LD, Viana ALd'A, Machado CV, Albuquerque MV, Oliveira RG, Iozzi FL, et al. Regionalização e acesso à saúde nos estados brasileiros: condicionantes históricos e político-institucionais. Ciênc Saúde Colet 2012; 17:2881-92.

4. Albuquerque MV, Viana ALd'A, Lima LD, Ferreira MP, Fusaro ER, Iozzi FL. Desigualdades regionais na saúde: mudanças observadas no Brasil de 2000 a 2016. Ciênc Saúde Colet 2017; 22:1055-64.

5. Araujo JD. Polarização epidemiológica no Brasil. Epidemiol Serv Saúde 2012; 21:533-8.

6. Freese E, Fontbonne A. Transição epidemiológica comparada: modernidade, precariedade e vulnerabilidade. In: Freese E, organizador. Epidemiologia, políticas e determinantes das doenças crônicas não transmissíveis no Brasil. Recife: Editora Universitária da UFPE; 2006. p. 17-45.

7. Frenk J, Frejka T, Bobadolla JL, Stern C, Lozano R, Sepúlveda JJ, et al. La transición epidemiológica en América Latina. Bol Ofic Sanit Panam 1991; 111:485-96.

8. Ministério da Saúde. Portaria no 1.378, de 9 de julho de 2013. Regulamenta as responsabilidades e define diretrizes para execução e financiamento das ações de Vigilância em Saúde pela União, Estados, Distrito Federal e Municípios, relativos ao Sistema Nacional de Vigilância em Saúde e Sistema Nacional de Vigilância Sanitária. Diário Oficial da União 2013; 10 jul. 
9. Conselho Nacional de Saúde. Resolução no 588, de 12 de julho de 2018. Institui a Política Nacional de Vigilância em Saúde (PNVS). Brasília: Conselho Nacional de Saúde; 2018.

10. Teixeira MG, Costa MCN, Carmo EH, Oliveira WK, Penna GO. Vigilância em Saúde no SUS - construção, efeitos e perspectivas. Ciênc Saúde Colet 2018; 23:1811-8.

11. Ministério da Saúde. Portaria no 4.279, de 30 de dezembro de 2010. Estabelece diretrizes para a organização da Rede de Atenção à Saúde no âmbito do Sistema Único de Saúde (SUS). Diário Oficial da União 2010; $30 \mathrm{dez}$.

12. Ministério da Saúde. Decreto no 7.508 , de 28 de junho de 2011. Regulamenta a Lei no 8.080, de 19 de setembro de 1990, para dispor sobre a organização do Sistema Único de Saúde SUS, o planejamento da saúde, a assistência à saúde e a articulação interfederativa e dá outras providências. Diário Oficial da União 2011; 29 jun.

13. Ministério da Saúde. Política Nacional de Vigilância em Saúde - documento base. http:// www.saude.sp.gov.br/resources/ccd/homepa ge/acesso-rapido/gpa/pnvs_2014_documen to_base.pdf (acessado em 27/Fev/2017).

14. Silva Júnior JB. Epidemiologia em serviço: uma avaliação de desempenho do sistema nacional de vigilância em saúde [Tese de Doutorado]. Campinas: Universidade Estadual de Campinas; 2004.

15. Teixeira GM, Costa MCN, Souza LP, Nascimento EMR, Barreto ML, Barbosa N, et al. Evaluation of Brazil's public health surveillance system within the context of the international health regulations. Rev Panam Salud Pública 2012; 32:49-55.

16. Albuquerque AC, Mota ELA, Felisberto E. Descentralização das ações de vigilância epidemiológica em Pernambuco, Brasil. Cad Saúde Pública 2015; 31:861-73.

17. Santos SSBS, Melo CMM, Costa HOG, Tanaka OY, Ramos FM, Santana MCC, et al. Avaliação da capacidade de gestão descentralizada da vigilância epidemiológica no Estado da Bahia. Ciênc Saúde Colet 2012; 17:873-82.

18. Oliveira CM, Casanova AO. Vigilância da saúde no espaço de práticas da atenção básica. Ciênc Saúde Colet 2009; 14:929-36.

19. Costa JMBS, Cesse EAP, Samico IC, Carvalho EMF. Avaliação do desempenho estadual da vigilância em saúde de Pernambuco. Physis (Rio J.) 2015; 25:1141-63.

20. Contandriopoulos AP, Champagne F, Denis JL, Pineault R. A avaliação na área da saúde: conceitos e métodos. In: Brousselle A, Champagne F, Contandriopoulos A-P, organizadores. Avaliação: conceitos e métodos. Rio de Janeiro: Editora Fiocruz; 2011. p. 19-40.

21. Viacava F, Ugá MAD, Porto S, Laguardia J, Moreira RS. Avaliação de desempenho de sistemas de saúde: um modelo de análise. Ciênc Saúde Colet 2012; 17:921-34.
22. Costa JMBS, Silva VL, Samico IC, Cesse EAP. Desempenho de intervenções de saúde em países da América Latina: uma revisão sistemática. Saúde Debate 2015; 39:307-19.

23. Larhey R, Nielsen SB. Rethinking the relationship among monitoring, evaluation and results based management: observations from Canada. New Dir Eval 2013; 137:45-56.

24. Marchal B, Hoerée T, Silveira VC, Belle SV, Prashanth NS, Kegels G. Building on the EGIPSS performance assessment: the multipolar framework as a heuristic to tackle the complexity of performance of public service oriented health care organizations. BMC Public Health 2014; 14:378-91.

25. Arreaza AL, Moraes JC. Vigilância da saúde: fundamentos, interfaces e tendências. Ciênc Saúde Colet 2010; 15:215-28.

26. Oliveira CM, Cruz MM. Sistema de vigilância em saúde no Brasil: avanços e desafios. Saúde Debate 2015; 39:255-67.

27. Viana ALD’Á, Ferreira MP, Cutrim MA, Fusaro ER, Souza MR, Mourão L, et al. O Processo de regionalização no Brasil: influência das dimensões Política, Estrutura e Organização. Rev Bras Saúde Mater Infant 2017; 17 Suppl 1:S27-43.

28. Viana ALD, Bousquat A, Pereira APCM, Uchimura LYT, Albuquerque MV, Mota PHS, et al. Tipologia das regiões de saúde: condicionantes estruturais para a regionalização no Brasil. Saúde Soc 2015; 24:413-22.

29. Albuquerque AC, Cesse EAP, Felisberto E, Samico I, Uchimura LYT, Estima N. Regionalização da vigilância em saúde: uma proposta de avaliação de desempenho em uma região de saúde no Brasil. Rev Bras Saúde Mater Infant 2017; 17 Suppl 1:S135-51

30. Kessner DM, Kalk CE, Singer J. Assessing health quality: the case for tracers. $\mathrm{N}$ Engl J Med 1973; 288:189-94.

31. Garnelo L, Sousa ABL, Silva CO. Regionalização em saúde no Amazonas: avanços e desafios. Ciênc Saúde Colet 2017; 22:1225-34.

32. Fundação Nacional de Saúde. Projeto Vigisus. Brasília: Fundação Nacional de Saúde; 1998.

33. Ministério da Saúde. Portaria no 1.708, de 16 de agosto de 2013. Regulamenta o Programa de Qualificação das Ações de Vigilância em Saúde (PQAVS), com a definição de suas diretrizes, financiamento, metodologia de adesão e critérios de avaliação dos Estados, Distrito Federal e Municípios. Diário Oficial da União 2013; 17 ago.

34. Tauil PL. Urbanização e ecologia do dengue. Cad Saúde Pública 2001; 17 Suppl:99-102.

35. Andrade HS, Oliveira VC, Gontijo TL, Pessôa MTC, Guimarães EAA. Avaliação do Programa de Controle da Tuberculose: um estudo de caso. Saúde Debate 2017; 41(n.spe): 242-58.

36. Shimizu HE, Cruz MS, Bretas Jr. N, Schierholt SR, Ramalho WM, Ramos MC, et al. O protagonismo dos Conselhos de Secretários Municipais no processo de governança regional. Ciênc Saúde Colet 2017; 22:1131-40. 
37. Durovni B, Saraceni V, Puppin MS, Tassinari W, Cruz OG, Cavalcante S, et al. The impact of the Brazilian Family Health Strategy and the conditional cash transfer on tuberculosis treatment outcomes in Rio de Janeiro: an individual-level analysis of secondary data. J Public Health (Oxf) 2018; 40:e359-66.

38. Costa EA. Vigilância sanitária e proteção da saúde. In: Departamento de Gestão da Educação na Saúde, Secretaria de Gestão do Trabalho e da Educação na Saúde, Ministério da Sáude, organizador. Direito e saúde pública: coletânea de textos. v. 1. Brasília: Ministério da Saúde; 2003. p. 180-203. (Série E. Legislação e Saúde).

39. Mendes A, Louvison MCP, Ianni AMZ, Leite MG, Feuerwerker LCM, Tanaka OU, et al. O processo de construção da gestão regional da saúde no Estado de São Paulo: subsídios para a análise. Saúde Soc 2015; 24:423-37.
40. Ministério da Saúde. Portaria no 3.992, de 28 de dezembro de 2017. Altera a Portaria de Consolidação no 6/GM/MS, de 28 de setembro de 2017, para dispor sobre o financiamento e a transferência dos recursos federais para as ações e os serviços públicos de saúde do Sistema Único de Saúde. Diário Oficial da União 2017; 28 dez.

41. Viana ALd'A, Bousquat A, Melo GA, De Negri Filho A, Medina MG. Regionalização e redes de saúde. Ciênc Saúde Colet 2018; 23:1791-8. 


\section{Abstract}

This study aimed to assess the performance of health surveillance regionalization with different levels of development in Brazil. A cross-sectional quantitative approach was used with data collected through a structured questionnaire, prepared on the basis of three study dimensions (Policy, Structure, and Organization), applied to 31 key actors in health surveillance in the selected Health Regions and municipalities. The measure of central tendency was mean score, and synthetic indices were obtained for each dimension and attribute. Three cutoff points were assigned to assess performance: values less than or equal to 4.99 were considered unsatisfactory; from 5.00 to 6.99, intermediate; and greater than or equal to 7.00, satisfactory. The study found that regionalization performance in health surveillance was only satisfactory in one region, intermediate in four, and unsatisfactory in one. Among the three dimensions, Policy and Organization showed the best performance and Structure showed the worst. In conclusion, in general, the higher the level of socioeconomic development and services supply, the better the performance in regionalization of health surveillance. The evaluation portrays the complexity of contexts in different regions of Brazil, thus contributing to understanding the dynamics of health surveillance regionalization in Brazil.

Regional Health Planning; Public Health Surveillance; Health Evaluation

\section{Resumen}

El objetivo de este estudio fue evaluar el desempeño de la regionalización de la vigilancia en salud en seis Regiones de Salud con diferentes niveles de desarrollo en Brasil. Se adoptó un abordaje cuantitativo transversal y los datos se recogieron utilizando un cuestionario estructurado, elaborado en base a tres dimensiones de investigación (Política, Estructura y Organización), aplicado a 31 actores-clave de la vigilancia en salud en las Regiones de Salud y en los municipios seleccionados. Se utilizó, como tendencia central, las puntuaciones promedio, y para cada dimensión y atributo se construyeron indices sintéticos. Se atribuyeron tres puntos de corte para la evaluación del desempeño: valores iguales o por debajo de 4,99, se consideraron insatisfactorios; entre 5,00 y 6,99, intermedios; e iguales o por encima de 7,00, satisfactorios. El estudio evidenció que el desempeño de la regionalización de la vigilancia en salud se consideró satisfactorio solamente en una región, intermedio en cuatro e insatisfactorio también en una. Entre las tres dimensiones, los mejores desempeños fueron los de Politica y Organización, y el más frágil, el de Estructura. Se concluye que, en general, cuanto mayor sea el nivel de desarrollo socioeconómico $y$ de oferta de servicios, mejor es el desempeño de la regionalización de la vigilancia en salud. La evaluación presentada retrata la complejidad de contextos de las diferentes regiones brasileñas, lo que contribuye a la comprensión de la dinámica de regionalización de la vigilancia en salud en el país.

Regionalización; Vigilancia en Salud Pública; Evaluación en Salud
Recebido em 30/Mar/2018

Versão final reapresentada em 06/Ago/2018 Aprovado em 01/Out/2018 